\title{
ADHESION and THIN-FILM MODULE RELIABILITY
}

\section{T.J. McMahon and G.J. Jorgensen National Renewable Energy Laboratory, Golden, CO 80401}




\section{Disclaimer and Government License}

This work has been authored by Midwest Research Institute (MRI) under Contract No. DE-AC36-99G010337 with the U.S. Department of Energy (the "DOE"). The United States Government (the "Government") retains and the publisher, by accepting the work for publication, acknowledges that the Government retains a non-exclusive, paid-up, irrevocable, worldwide license to publish or reproduce the published form of this work, or allow others to do so, for Government purposes.

Neither MRI, the DOE, the Government, nor any other agency thereof, nor any of their employees, makes any warranty, express or implied, or assumes any liability or responsibility for the accuracy, completeness, or usefulness of any information, apparatus, product, or process disclosed, or represents that its use would not infringe any privately owned rights. Reference herein to any specific commercial product, process, or service by trade name, trademark, manufacturer, or otherwise does not constitute or imply its endorsement, recommendation, or favoring by the Government or any agency thereof. The views and opinions of the authors and/or presenters expressed herein do not necessarily state or reflect those of MRI, the DOE, the Government, or any agency thereof. 


\section{Outline}

We measure the weakest adhesion between layers of various thin-film (T-F) modules/samples.

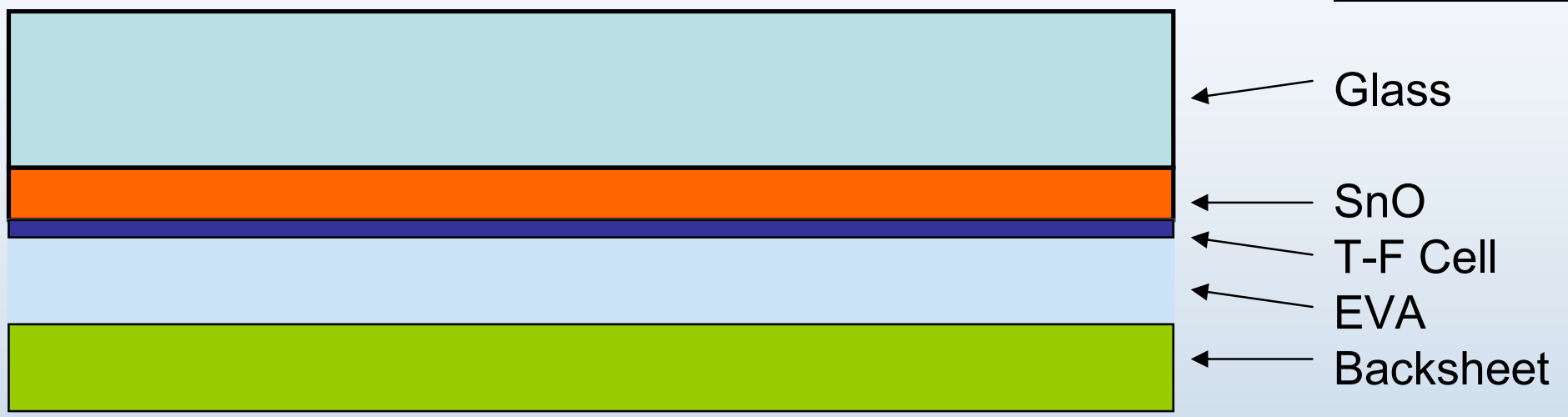

\section{$\underline{\text { A-Si or CIGS }}$}

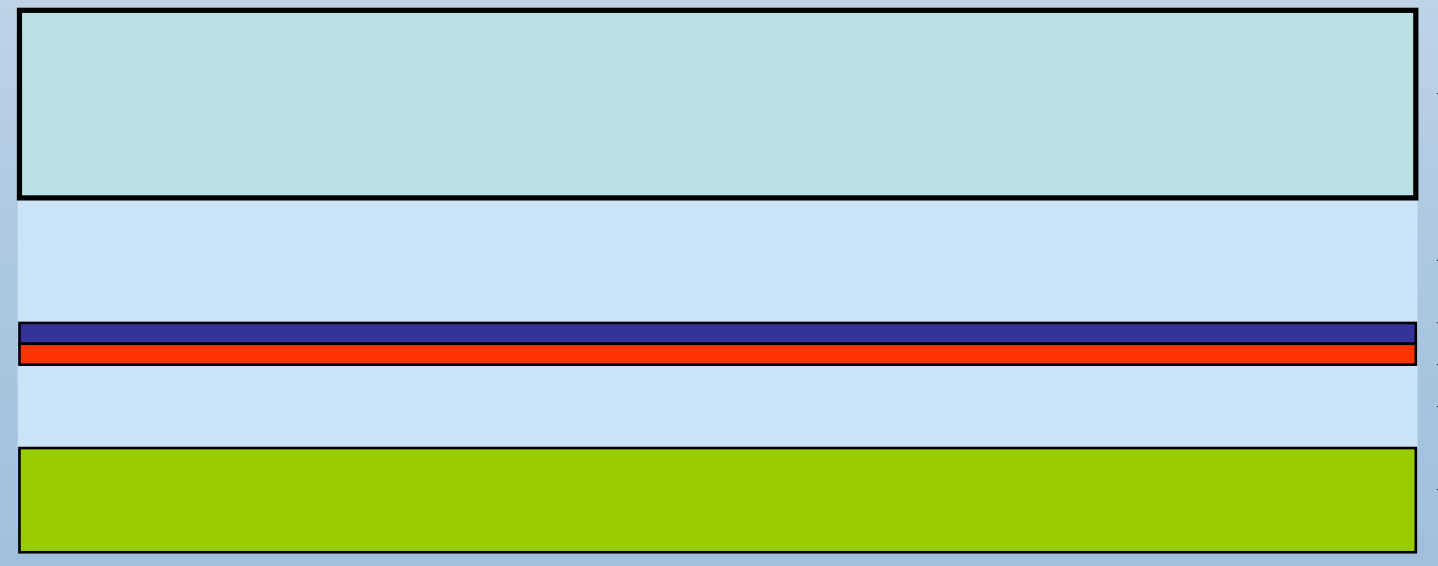

$\longleftarrow$

\section{Glass/ETFE}

\section{EVA}

T-F Cell

SS/glass

EVA

Backsheet 


\section{Outline}

- We measure the weakest interlayer adhesion between layers of various thin-film (T-F) modules/samples.

- An Instron mechanical testing unit is used to measure peel strengths at $90^{\circ}$ or $180^{\circ}$ on laminations of three T-F technologies,

- before and after damp heat / UV

- and at elevated temperatures.

- ASTM D3359 - scratch and tape pull test to evaluate cell adhesion strengths. 


\section{Outline}

- We measure the weakest interlayer adhesion between layers of various thin-film (T-F) modules/samples.

- An Instron mechanical testing unit is used to measure peel strengths at $90^{\circ}$ or $180^{\circ}$ on laminations of three T-F technologies,

- before and after damp heat / UV

- in one instance at elevated temperatures.

- In some cases, a scratch and tape pull test to evaluate inter-cell layer adhesion strengths.

- Measure effect of higher temperature and relative humidity $(\mathrm{RH})$ adhesion/cohesion. 


\section{Outline}

- We measure the weakest interlayer adhesion between layers of various thin-film (T-F) modules/samples.

- An Instron mechanical testing unit is used to measure peel strengths at $90^{\circ}$ or $180^{\circ}$ on laminations of three T-F technologies,

- cell contact layers to glass,

- contact layers to the semiconductor,

- encapsulant to cell, glass, or backsheet, etc.

- before and after damp heat / UV

- in one instance at elevated temperatures.

- In some cases, a scratch and tape pull test to evaluate inter-cell layer adhesion strengths.

- Effect of higher temperature and relative humidity (RH) adhesion/cohesion.

- Adhesion's effect on thin-film (T-F) module reliability. 


\section{$\mathrm{SnO}_{2}$ Delamination}

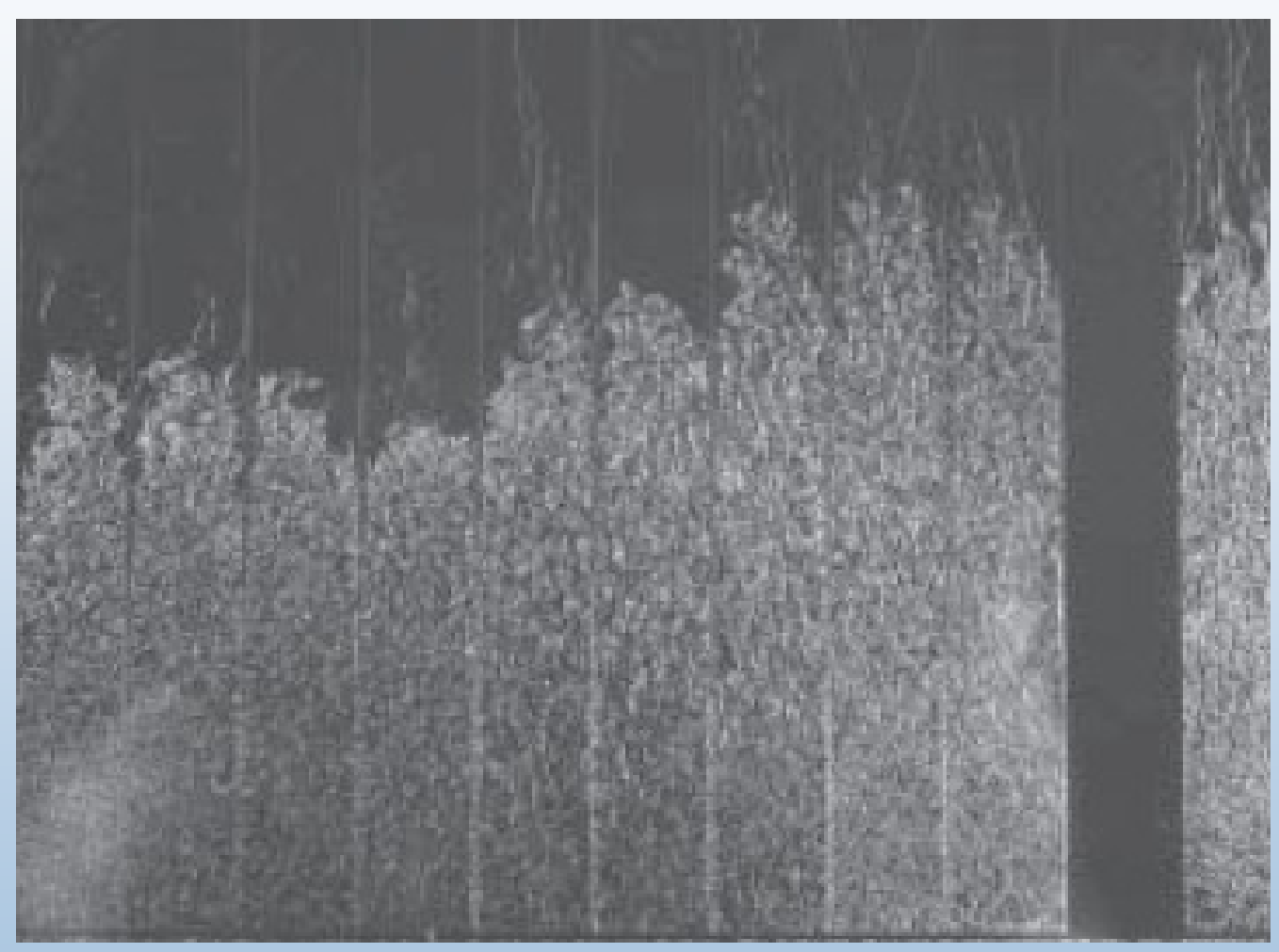

Cause: Heat, humidity, and high voltage, 


\section{Bubble-type Delamination}

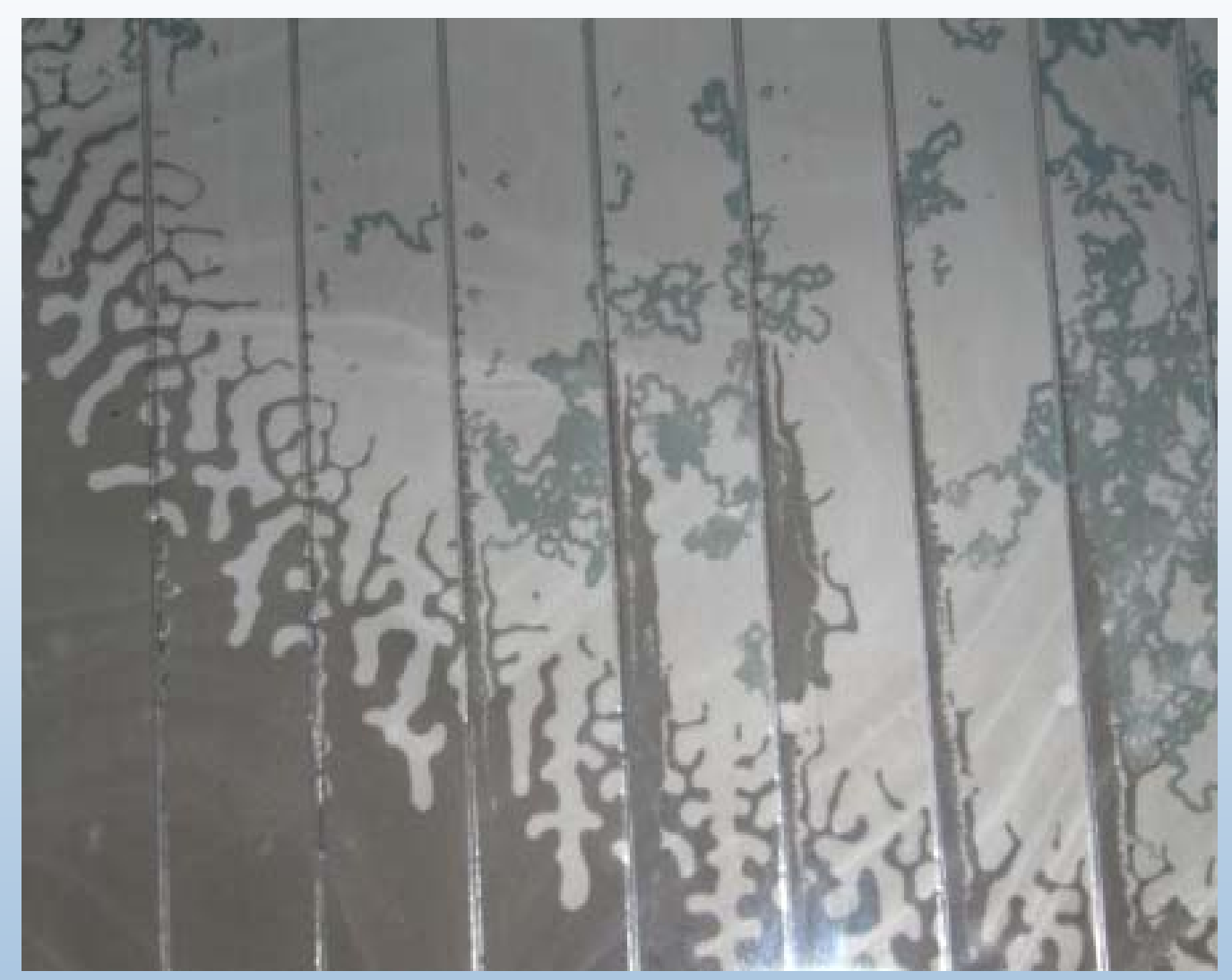

Cause: Heat and tensile stress. 


\section{Adhesion Pull Samples}
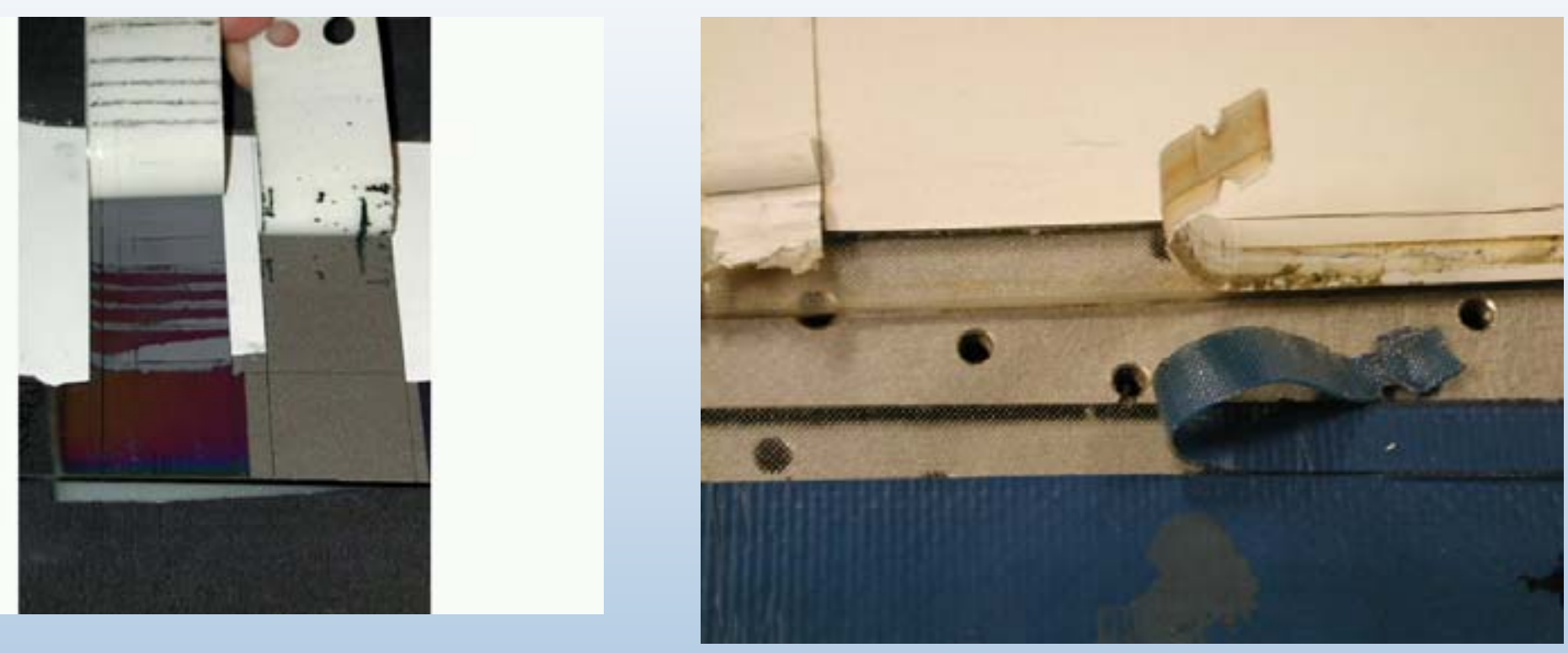


\section{Instron Peel Test}
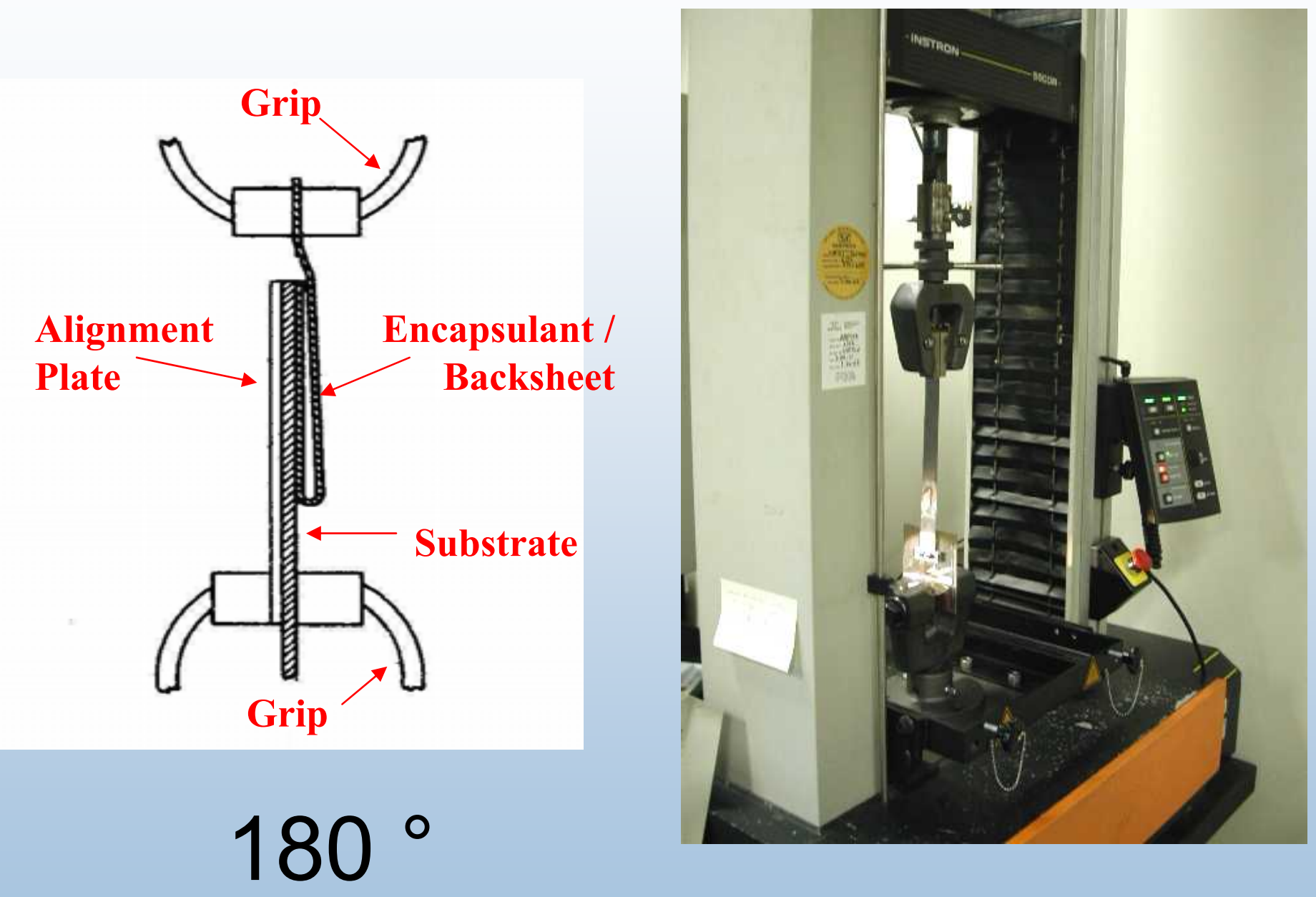


\section{Adhesion Strength Table}

\begin{tabular}{|c|c|c|c|c|}
\hline \multirow[b]{2}{*}{$\begin{array}{l}\text { Device: } \\
\text { Source }\end{array}$} & \multirow{2}{*}{$\begin{array}{c}\text { Failure } \\
\text { Interface }\end{array}$} & \multicolumn{2}{|c|}{ Weathering } & \multirow{2}{*}{$\begin{array}{c}\text { Peel } \\
\text { Strength } \\
(\mathrm{N} / \mathrm{mm})\end{array}$} \\
\hline & & Time & Type & \\
\hline \multirow{2}{*}{$\begin{array}{c}\mathrm{a}-\mathrm{Si}: \\
\mathrm{A}\end{array}$} & $\mathrm{EVA} / \mathrm{a}-\mathrm{Si}$ & 0 & none & 3.8 \\
\hline & EVA / a-Si & $92 \mathrm{~h}$ & $85 / 85$ & 3.1 \\
\hline \multirow{2}{*}{$\begin{array}{c}\text { SiOxNy / } \\
\text { Ni paste/ } \\
\text { Graphite/ } \\
\text { CdTe: B }\end{array}$} & EVA / SiOxNy & 0 & none & 5.6 \\
\hline & $\begin{array}{c}\mathrm{SiOxNy} / \mathrm{Ni} \\
\text { paste }\end{array}$ & $256 \mathrm{~h}$ & $85 / 85$ & 4.5 \\
\hline
\end{tabular}

3-h dry-out after removal from $85^{\circ} \mathrm{C} / 85 \% \mathrm{RH}$ 


\section{Adhesion Strength Table}

\begin{tabular}{|c|c|c|c|c|}
\hline \multirow[b]{2}{*}{$\begin{array}{l}\text { Device: } \\
\text { Source }\end{array}$} & \multirow[b]{2}{*}{$\begin{array}{c}\text { Failure } \\
\text { Interface }\end{array}$} & \multicolumn{2}{|c|}{ Weathering } & \multirow{2}{*}{$\begin{array}{c}\text { Peel } \\
\text { Strength } \\
(\mathrm{N} / \mathrm{mm})\end{array}$} \\
\hline & & Time & Type & \\
\hline \multirow{2}{*}{$\begin{array}{c}\text { a-Si: } \\
\text { A }\end{array}$} & EVA / a-Si & 0 & none & 3.8 \\
\hline & EVA / a-Si & $92 \mathrm{~h}$ & $85 / 85$ & 3.1 \\
\hline \multirow{2}{*}{$\begin{array}{c}\text { SiOxNy / } \\
\text { Ni paste/ } \\
\text { Graphite/ } \\
\text { CdTe: B }\end{array}$} & EVA / SiOxNy & 0 & none & 5.6 \\
\hline & $\begin{array}{l}\mathrm{SiOxNy} / \mathrm{Ni} \\
\text { paste }\end{array}$ & $256 \mathrm{~h}$ & $85 / 85$ & 4.5 \\
\hline \multirow{3}{*}{$\begin{array}{l}\text { SiOxNy / } \\
\text { CdTe: B }\end{array}$} & EVA / SiOxNy & 0 & none & 7.0 \\
\hline & $\mathrm{SiOxNy} / \mathrm{CdTe}$ & 0 & none & 2.0 \\
\hline & $\mathrm{SiOxNy} / \mathrm{CdTe}$ & $256 \mathrm{~h}$ & $85 / 85$ & 0.3 \\
\hline \multirow{2}{*}{$\begin{array}{l}\text { SiOxNy / } \\
\text { CdTe: C }\end{array}$} & EVA / SiOxNy & 0 & none & 6.4 \\
\hline & EVA / SiOxNy & 256 & $85 / 85$ & 4.9 \\
\hline CdTe: C & $\begin{array}{c}\text { EVA / Metal- } \\
\text { CdTe }\end{array}$ & 0 & none & 1.1 \\
\hline CdTe: C & $\begin{array}{c}\text { EVA / Metal- } \\
\text { CdTe } \\
\end{array}$ & 256 & $85 / 85$ & 0.6 \\
\hline $\begin{array}{l}\text { CdTe: C } \\
\text { (left) }\end{array}$ & $\begin{array}{c}\text { EVA / Metal- } \\
\text { CdTe }\end{array}$ & 0 & none & 1.0 \\
\hline (middle) & $\begin{array}{c}\text { EVA / Metal- } \\
\text { CdTe } \\
\end{array}$ & 0 & none & 0.7 \\
\hline (right) & Metal / CdTe & 0 & none & 0.06 \\
\hline CIGS:D1 & CIGS / Mo & 0 & none & 0.05 \\
\hline CIGS:D2 & EVA / CIGS & 0 & & 7.0 \\
\hline $60 \mathrm{C}$ & EVA / CIGS & 0 & none none & 1.1 \\
\hline $80 \mathrm{C}$ & EVA cohesive & 0 & none & 0.05 \\
\hline
\end{tabular}

\begin{tabular}{|c|c|c|c|c|}
\hline \multirow[b]{2}{*}{$\begin{array}{l}\text { Device: } \\
\text { Source }\end{array}$} & \multirow[b]{2}{*}{$\begin{array}{l}\text { Failure } \\
\text { Interface }\end{array}$} & \multicolumn{2}{|c|}{ Weathering } & \multirow{2}{*}{$\begin{array}{c}\text { Peel } \\
\text { Strength } \\
(\mathrm{N} / \mathrm{mm}) \\
\end{array}$} \\
\hline & & Time & Type & \\
\hline CIGS: E & EVA / CIGS & 0 & none & 0.9 \\
\hline CIGS & CIGS / Mo & 0 & none & 0.3 \\
\hline CIGS & EVA / CIGS & $258 \mathrm{~h}$ & $85 / 85$ & 0.6 \\
\hline CIGS & Tefzel / EVA & $258 \mathrm{~h}$ & $85 / 85$ & 0.02 \\
\hline CIGS & Stainless / EVA & $258 \mathrm{~h}$ & $85 / 85$ & 0.5 \\
\hline CIGS & EVA / CIGS & $7 \mathrm{mo}$ & Cocoa, FL & 0.8 \\
\hline CIGS & EVA / CIGS & $16 \mathrm{mo}$ & $\begin{array}{c}\text { Golden, } \\
\text { CO }\end{array}$ & 0.9 \\
\hline CIGS & Stainless / EVA & $16 \mathrm{mo}$ & $\begin{array}{c}\text { Golden, } \\
\text { CO }\end{array}$ & 0.8 \\
\hline \multirow{2}{*}{$\begin{array}{c}\text { TPE/EVA/ } \\
\text { Glass: F } \\
\text { control } \\
\end{array}$} & EVA / Glass & 0 & none & 5.5 \\
\hline & EVA / Glass & $16 \mathrm{~h}$ & $85 / 85$ & 3.8 \\
\hline \multirow{2}{*}{$\begin{array}{c}\text { TPE/EVA/ } \\
\text { Glass: F } \\
\text { exposed }\end{array}$} & EVA / Glass & $\begin{array}{l}7 \mathrm{yr} \\
\text { equal }\end{array}$ & UV lamp & 2.0 \\
\hline & EVA / Glass & $+16 \mathrm{~h}$ & $85 / 85$ & 1.5 \\
\hline \multirow{2}{*}{$\begin{array}{c}\text { Tedlar/EVA/ } \\
\text { Glass: G } \\
\text { control } \\
\end{array}$} & EVA / Glass & 0 & none & 3.2 \\
\hline & EVA / Glass & $17 \mathrm{~h}$ & $85 / 85$ & 1.8 \\
\hline \multirow{2}{*}{$\begin{array}{c}\text { Tedlar/EVA/ } \\
\text { Glass: G } \\
\text { exposed }\end{array}$} & EVA / Glass & $\begin{array}{c}7 \mathrm{yr} \\
\text { equal }\end{array}$ & UV lamp & 0.4 \\
\hline & EVA / Glass & $+17 \mathrm{~h}$ & $85 / 85$ & 0.4 \\
\hline $\begin{array}{c}\text { Scotch } \\
\text { Tape/Glass }\end{array}$ & Tape / Glass & 0 & none & 0.08 \\
\hline $\begin{array}{c}\text { ASTM } \\
\text { Tape/Glass }\end{array}$ & Tape / Glass & 0 & none & 0.4 \\
\hline
\end{tabular}




\section{CdTe:B cell peel tested}

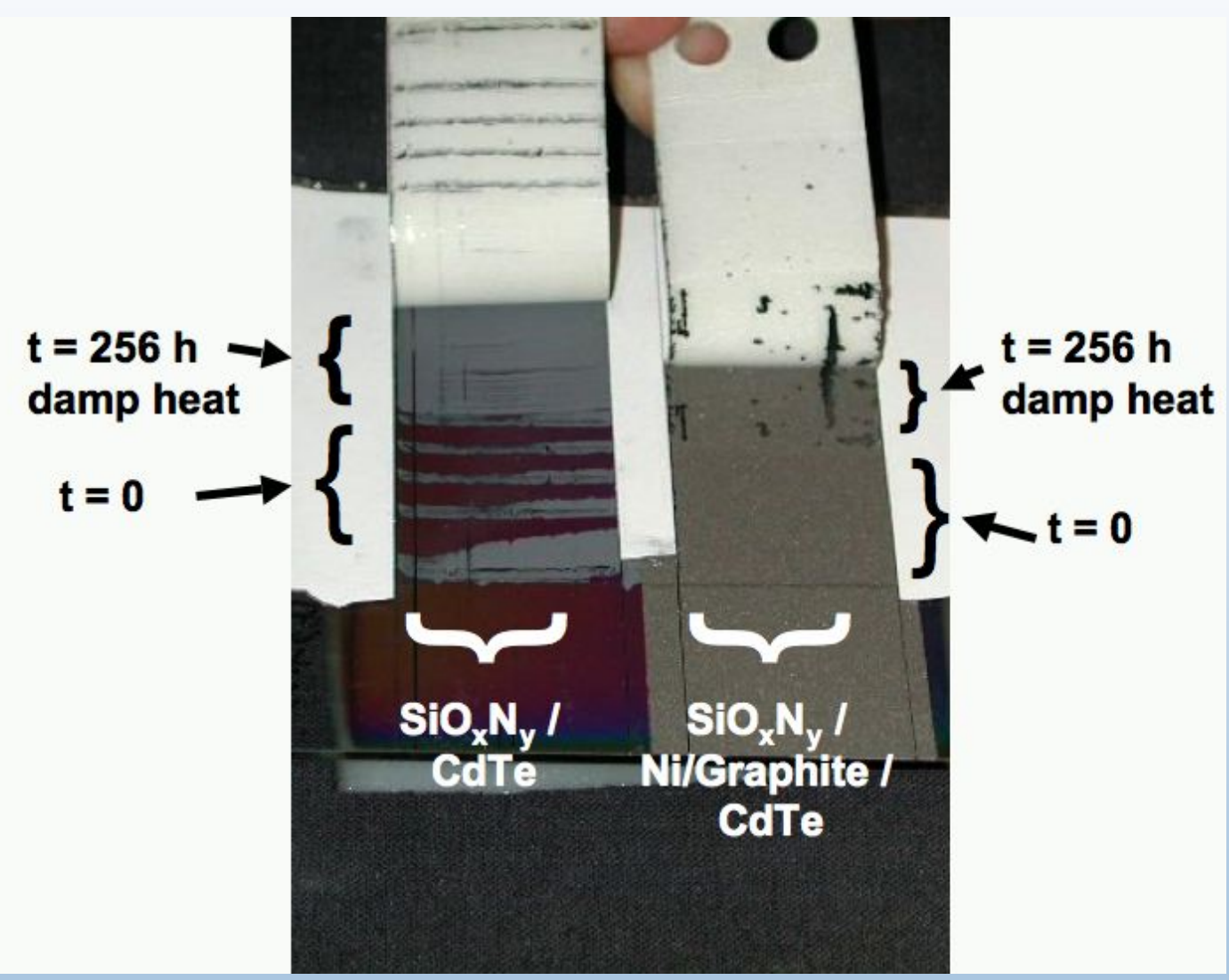




\section{Instron peel data: CdTe cell}

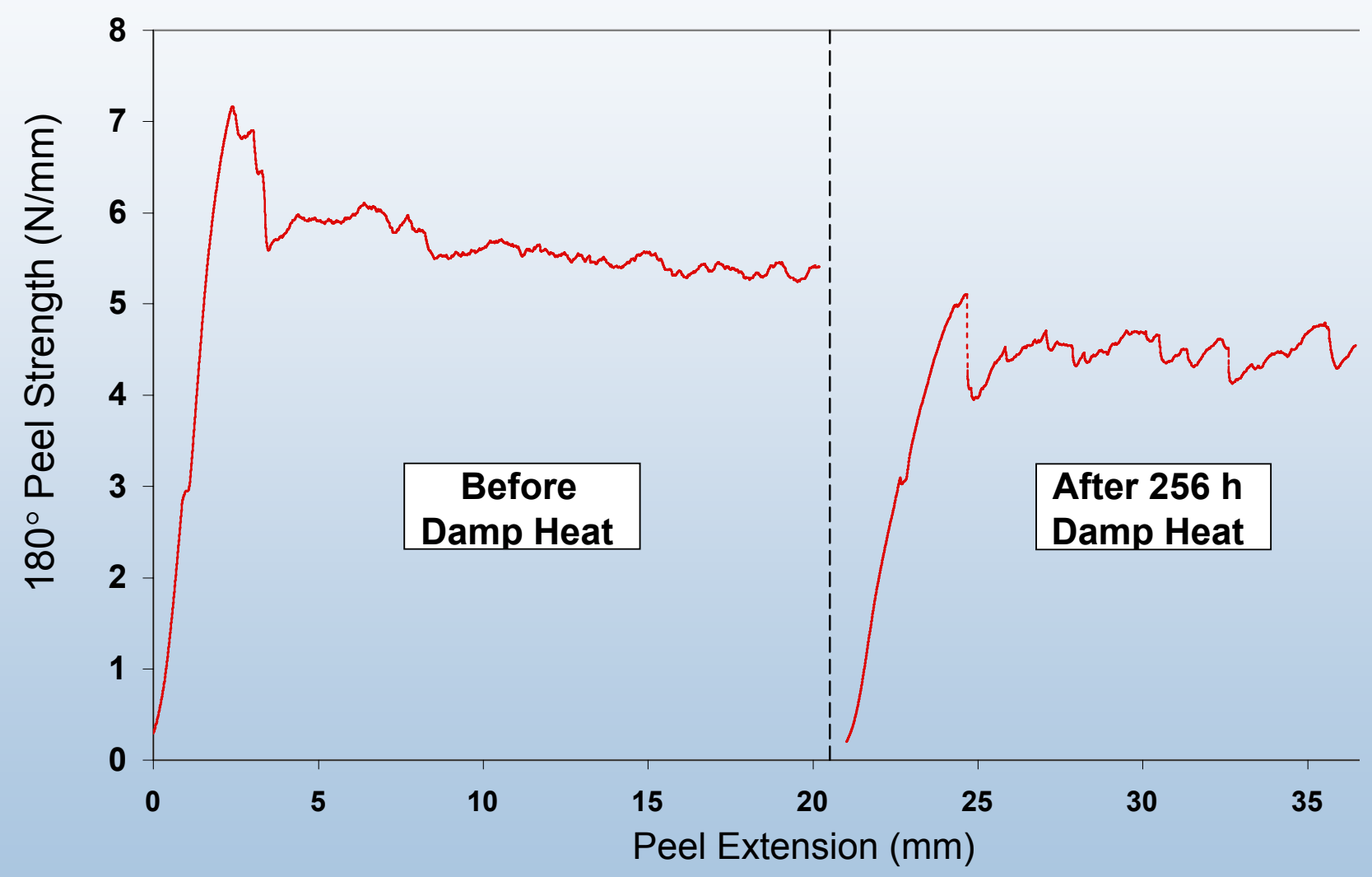




\section{Instron peels: two Si-modules}

F1, Control: 5.5 (N/mm)

G1, Control: $3.2(\mathrm{~N} / \mathrm{mm})$

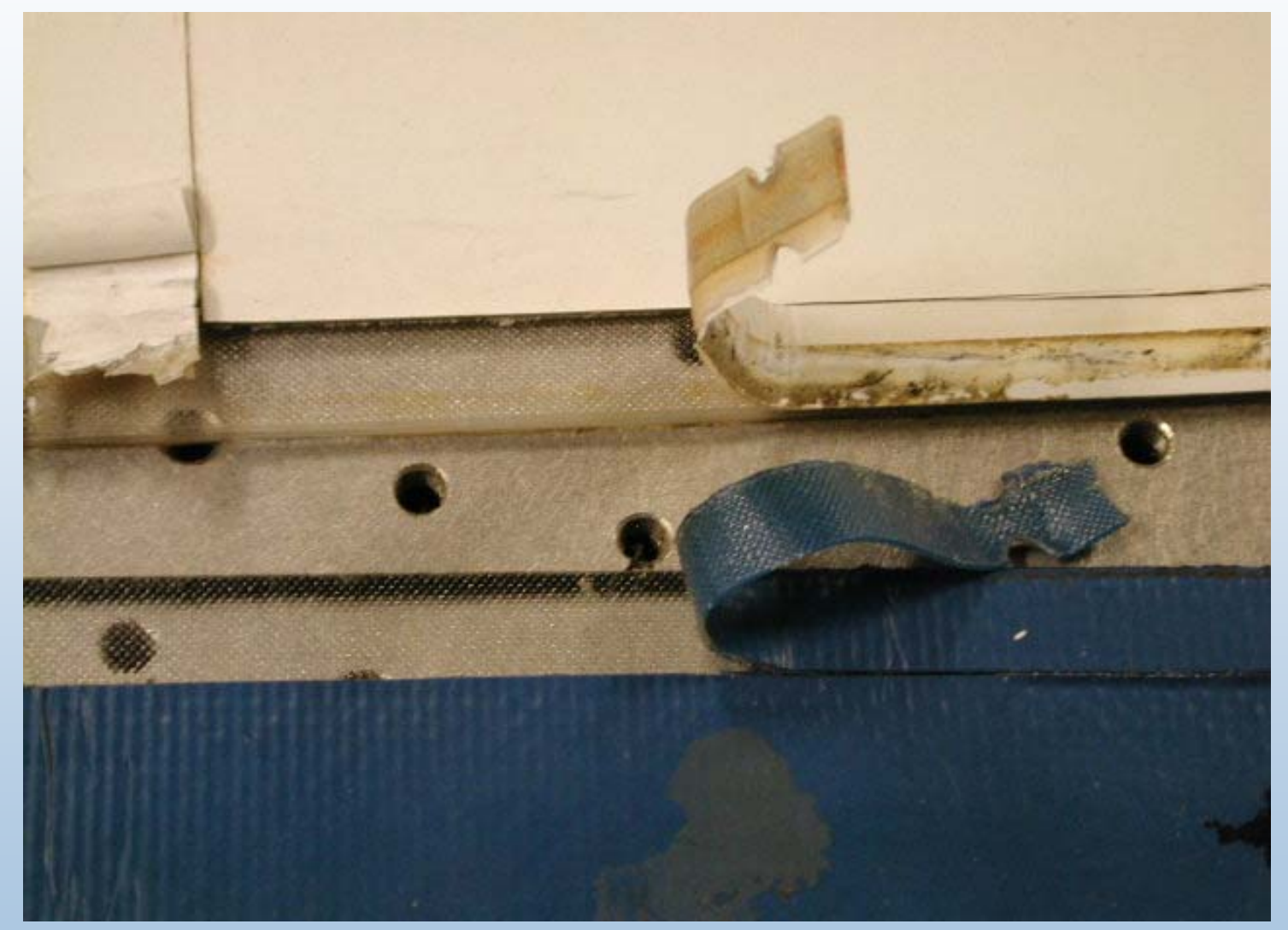




\section{Instron peels: two Si-modules}

F1, Control: 5.5 (N/mm)

F2, 7 yr UV: 2.0

G1, Control: $3.2(\mathrm{~N} / \mathrm{mm})$

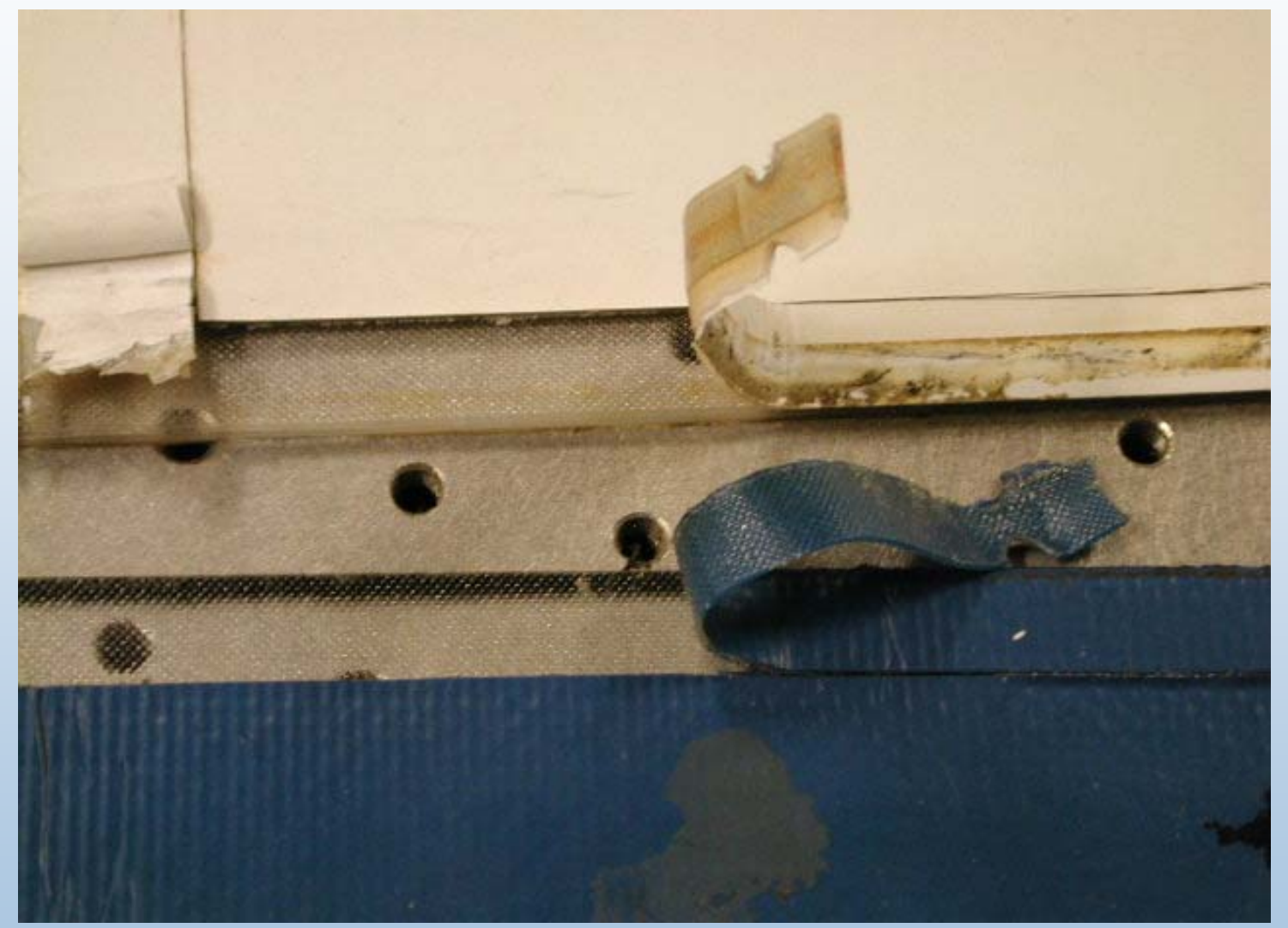

G2, 7 yr UV: 0.4 


\section{Instron peels: two Si-modules}

F1, Control: $5.5(\mathrm{~N} / \mathrm{mm})$ Plus 16h DH: 3.8

F2, 7 yr UV: 2.0 Plus 16h DH: 1.5

G1, Control: $3.2(\mathrm{~N} / \mathrm{mm})$ Plus 16h DH: 1.8

G2, 7 yr UV: 0.4 Plus 16h DH: 0.4

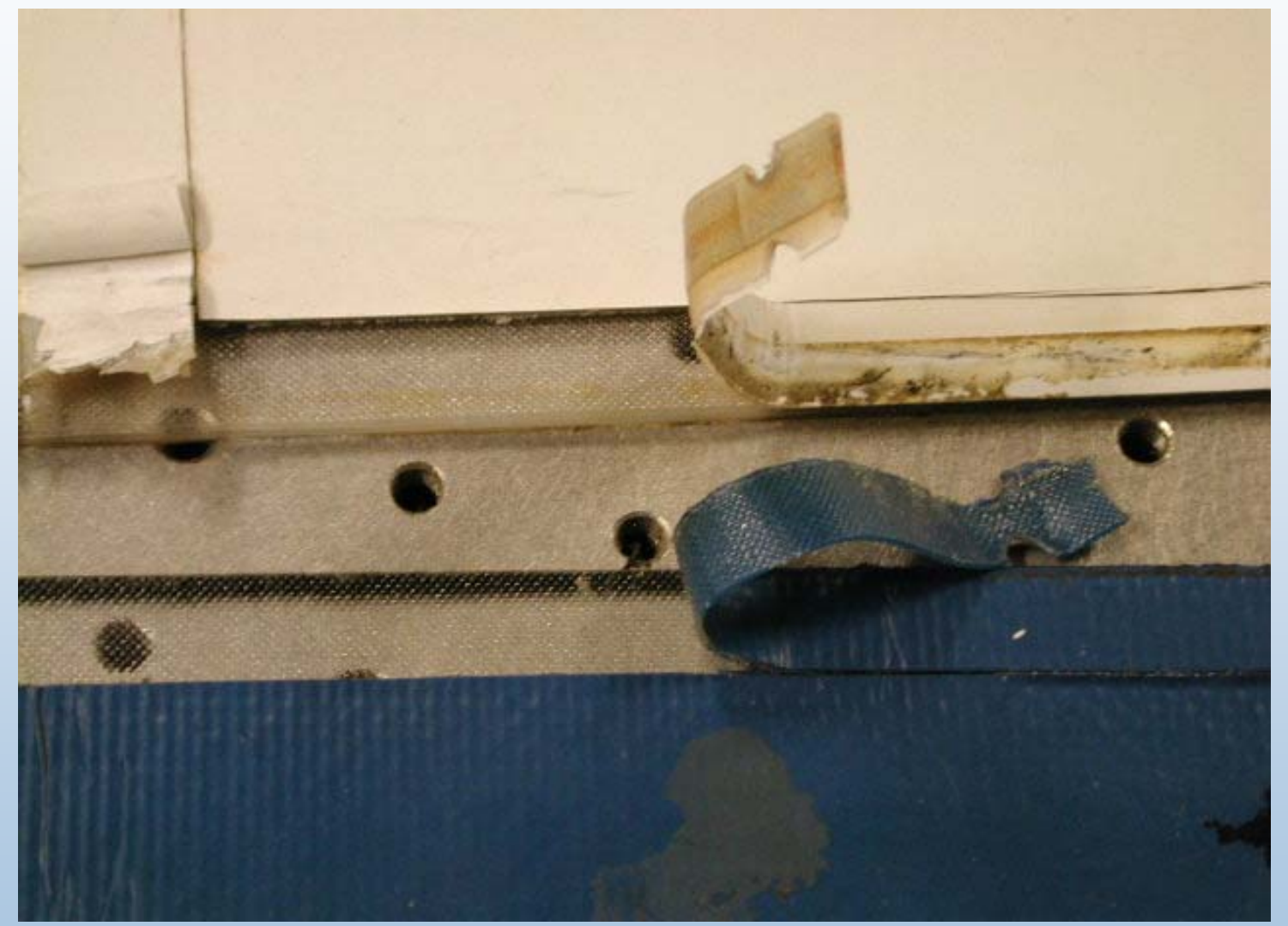




\section{CIGS:D2 De-encapsulation}

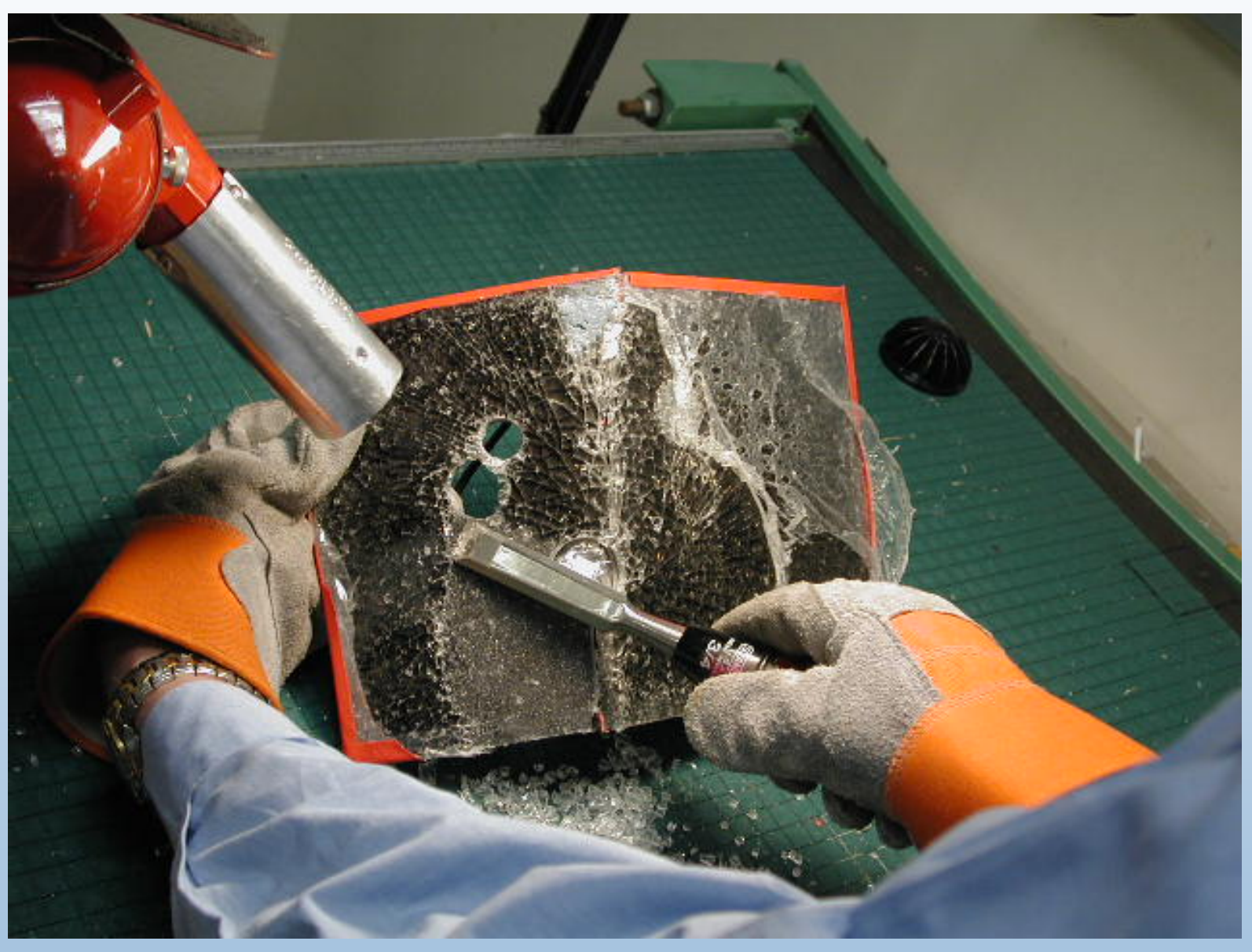




\section{EVA Peel Strength vs. Temperature}

\section{CIGS:D2}

\begin{tabular}{|c|c|c|}
\hline Temperature & Failure & $\begin{array}{c}180^{\circ} \text { Peel } \\
\text { Strength } \\
(\mathrm{N} / \mathrm{mm})\end{array}$ \\
$25^{\circ} \mathrm{C}$ & EVA / CIGS & 7.0 \\
$60^{\circ} \mathrm{C}$ & EVA / CIGS & 1.1 \\
$80^{\circ} \mathrm{C}$ & EVA cohesive & 0.05 \\
\hline
\end{tabular}




\section{Bubble-type Delamination}

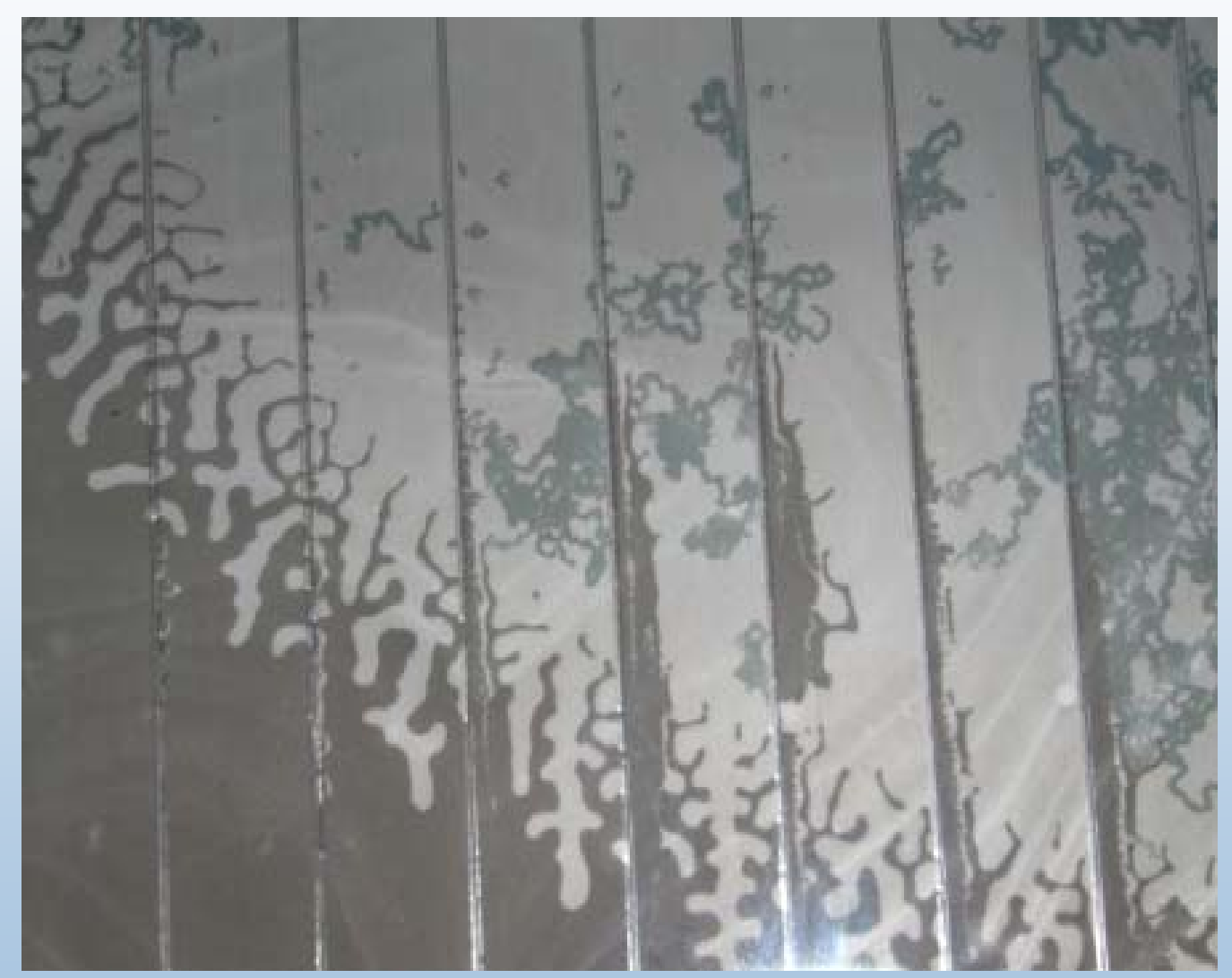




\section{ASTM D 3359-02 scratch test}

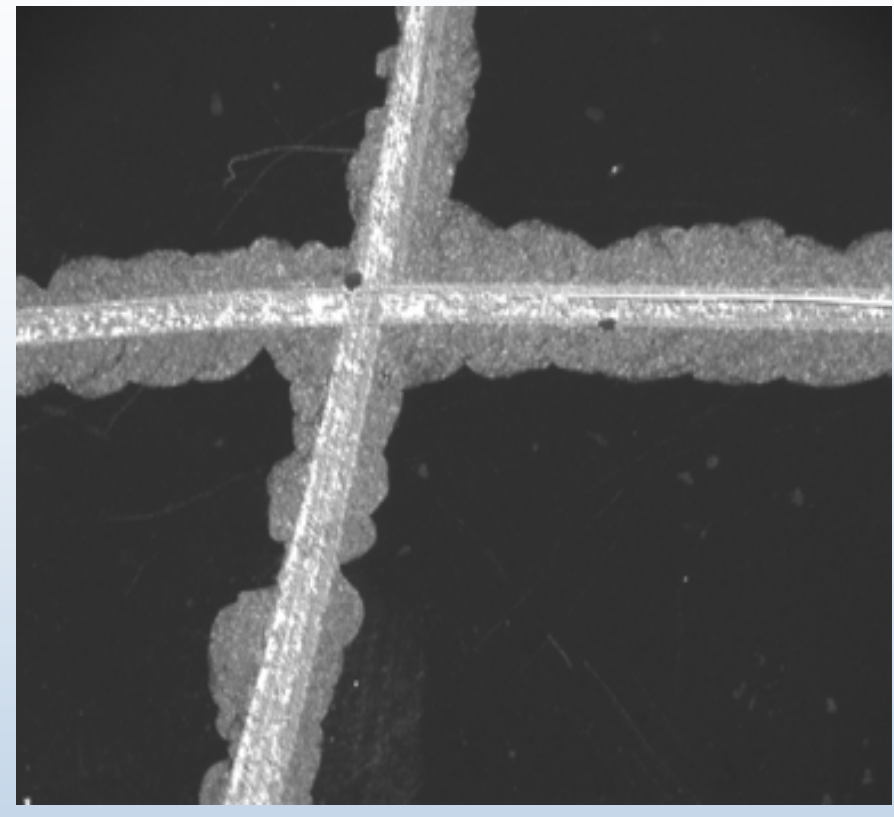

CIGS:D2

$>7 \mathrm{~N} / \mathrm{mm}$
A lattice pattern is scratched into the coated surface with six or seven lines in each direction.

Any loose fragments are brushed away.

The ASTM qualified tape is pressed firmly to that area.

Within 30 to $90 \mathrm{~s}$, the tape is pulled back at a $180^{\circ}$ angle.

The ASTM-designated tape would exert a force of 0.4 $\mathrm{N} / \mathrm{mm}$ on any cell material disturbed or loosened by the scratch 


\section{ASTM D 3359-02 scratch test}

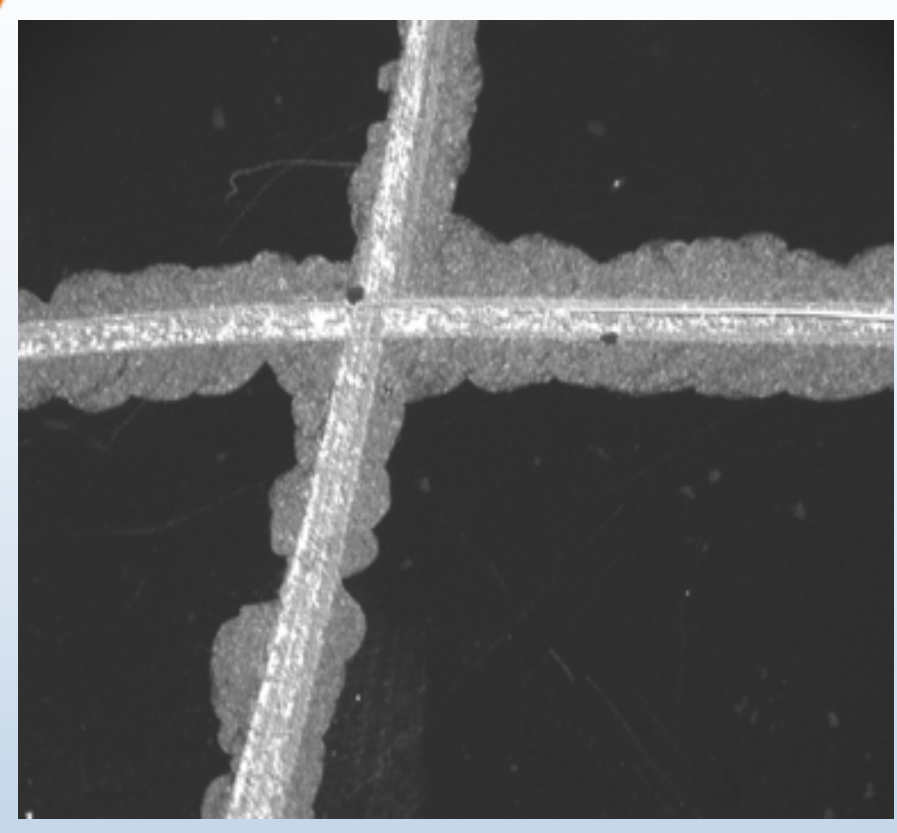

CIGS:D2

$>7 \mathrm{~N} / \mathrm{mm}$

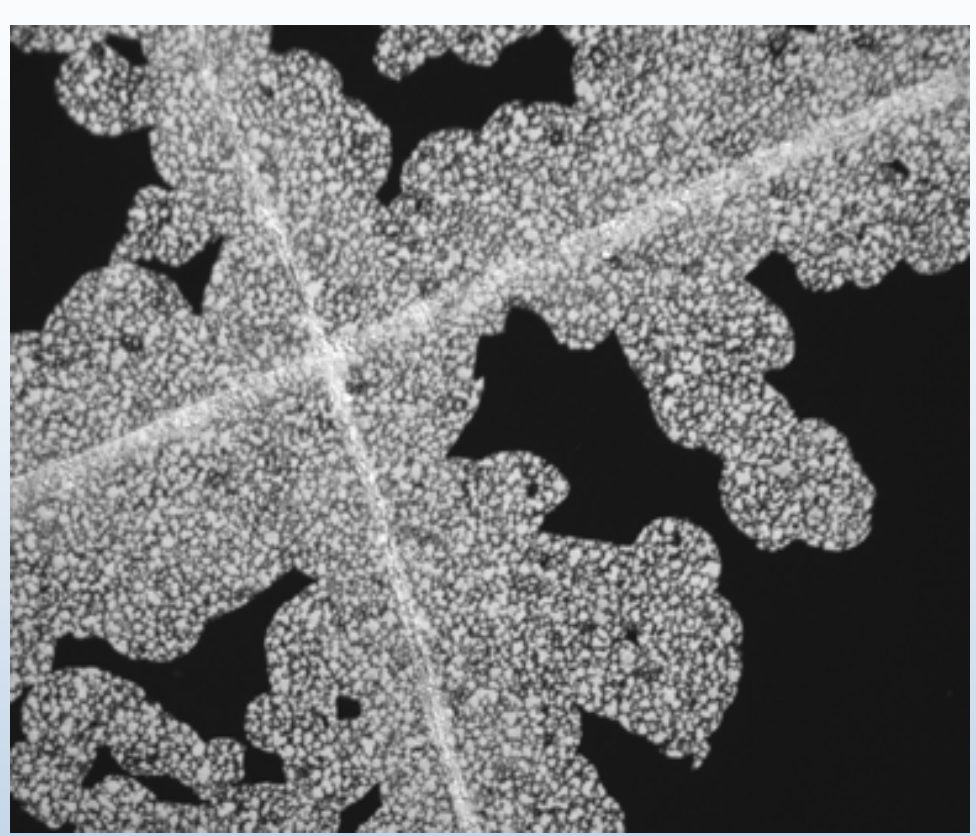

CIGS:D1

$0.05 \mathrm{~N} / \mathrm{mm}$ (CIGS/Mo)

Tarrant, D. E.; Gay, R. R. (1995). Research on High-Efficiency, Large-Area CulnSe $_{2}$-Based Thin-Film Modules: Final Subcontract Report, 16 August 1993 - 30 June 1995. 99 pp.; NREL Report No. TP-413-8121. (83014.PDF) 


\section{ASTM D 3359-02 scratch test}
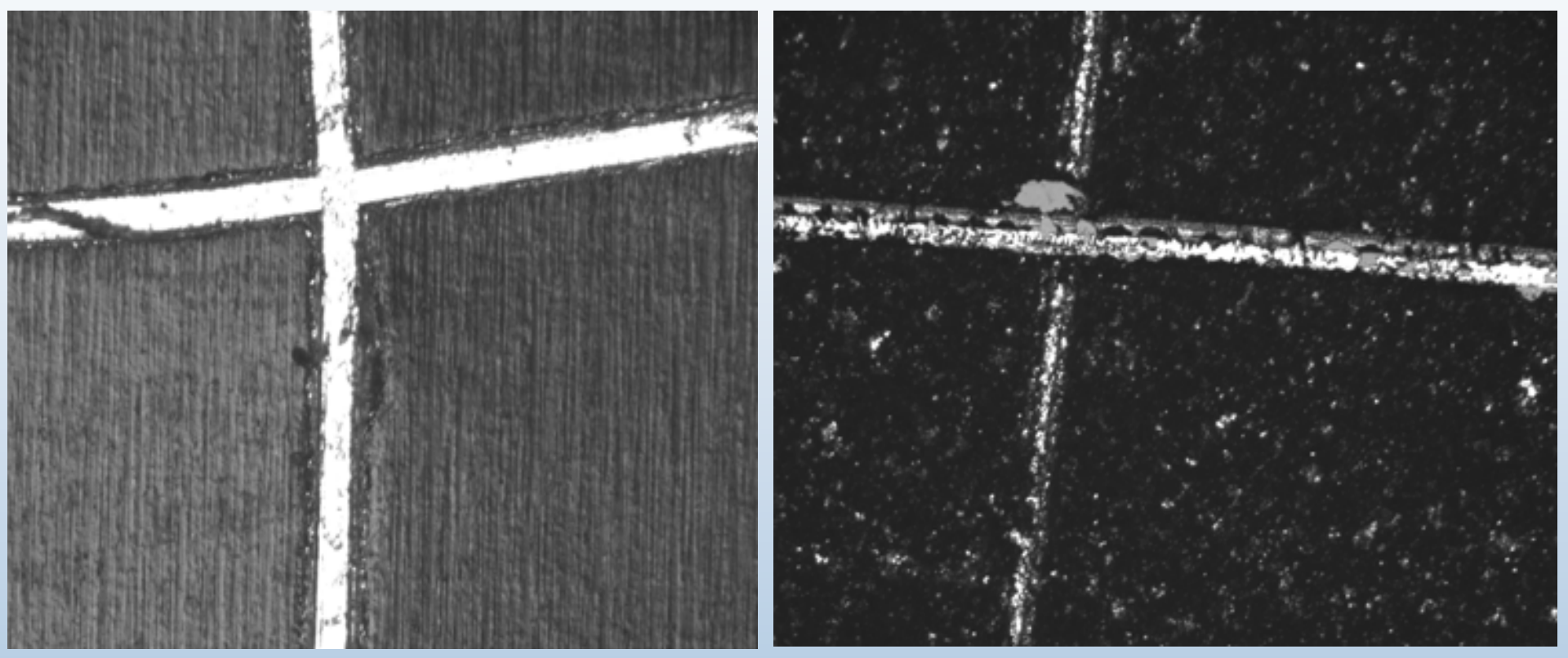

a-Si:A

$>3.8 \mathrm{~N} / \mathrm{mm}$
CdTe:B

$>5.6 \mathrm{~N} / \mathrm{mm}$ 


\section{Summary}

- Measured interface peel strength values of various T-F module technologies and how they are affected by environmental stress; some were quite low. 


\section{Summary}

- Measured interface peel strength values of various T-F module technologies and how they are affected by environmental stress; some were quite low.

- Adhesion at higher $\mathrm{T}$ and $\mathrm{RH}$, and after extended UV and $\mathrm{RH}$ exposure show a reduction in strength.

- Minimum adhesion strength defined at higher T and RH.

- The softening of EVA near $85^{\circ} \mathrm{C}$ can lead to failure. 


\section{Summary}

Measured interface peel strength values of various T-F module technologies and how they are affected by environmental stress; some were quite low.

Adhesion at higher $\mathrm{T}$ and $\mathrm{RH}$, and after extended $\mathrm{UV}$ and $\mathrm{RH}$ exposure show a reduction in strength.

A minimum adhesion strength defined at higher $\mathrm{T}$ and $\mathrm{RH}$.

The softening of EVA near $85^{\circ} \mathrm{C}$ can lead to failure.

\section{Achieve highest adhesion possible for corrosion and water ingress reduction.}




\section{Summary}

- Measured interface peel strength values of various T-F module technologies and how they are affected by environmental stress; some were quite low.

- Adhesion at higher T and $\mathrm{RH}$, and after extended UV and $\mathrm{RH}$ exposure show a reduction in strength.

- A minimum adhesion strength perhaps at higher $\mathrm{T}$ and $\mathrm{RH}$.

- The softening of EVA near $85^{\circ} \mathrm{C}$ can lead to failure.

- Achieve highest adhesion possible for corrosion and water ingress reduction.

- Interlayer adhesion in a T-F cell can be quite small and within a T-F technology the ASTM D 3359-02 "Measuring Adhesion by Tape Test" can be useful as a screening test. 